\title{
Adaptive Observer for Multi-Effectors Aircraft with Control Surface Damage
}

\author{
Shuang ZHANG
}

The First Aeronautical College of Air Force, Xinyang, 464000, China

Keywords: Multi-effectors aircraft, Control surface damage, Fault diagnosis. Adaptive compensation observer.

\begin{abstract}
An adaptive compensation observer is presented for fast diagnose of multi-effectors aircraft in the presence of control surfaces fault, which directly estimates the deflection angles of control surfaces. An augmented observer is designed for the estimation of system input, and an adaptive compensation is introduced to improve the dynamic tracking performance of observer. Simulation results suggest that, for different cases of actuator faults, the proposed observer methods can achieve fault alarm within 20 milliseconds and determine the fault location in 0.22 seconds.
\end{abstract}

\section{Introduction}

At present, the lack of rapid and effective control surface fault diagnosis method is a key factor restricting the development of Active Fault Tolerant Control aircraft[1,2]. As it relates to the entire closed-loop flight control system, damage control surface failure is one of the hot and difficult problems[3]. Although there is no published literature troubleshooting clear that more time is short to meet the requirements of reconfigurable control, but can be inferred that the aircraft should be 0.7 times within a short period[4], which made a great real-time challenge.

To develop active fault tolerant control distribution technology, it must solve the problem of realtime fault diagnosis, fault diagnosis of the current model-based approach rudder mainly, parameter identification, filter and observer methods. Parameter identification method is generally related derivative aircraft operated online identification, fault information obtained rudder surface. The recursive least square (RLS) was converted to the frequency domain by Girish Chowdhary[5]. The frequency domain least squares (FTR) to improve the accuracy of noise adaptability, can in no prior knowledge within two seconds of the estimated derivative manipulation, which was widely used in various fields[6]. The literature[7,8] is the use of real-time advantage RLS efficient, innovative application-specific on its basis in order to improve recognition precision diagnosis within 5 seconds, under the premise of a large number of discrete calculation up to 0.5 seconds. Although the parameter identification method is faster, it is a lot of off-line data and accurate fault aerodynamic parameters, as will as based on the extent of damage and aerodynamic data.

Therefore, to realize the rapid and effective fault diagnosis for control surface damage is still a problem. This paper focus on the rapidity and accuracy of fault diagnosis method. The major work includes:

Fast and accurate tracking problem in control surface state, proposed an adaptive compensation observer method. The method is based on the "enough to satisfy the real-time fault diagnosis method is simple" aircraft of the idea of [5], direct estimation of the rudder deflection by the augmented state variables, using the adaptive compensation control surface estimation method to solve the augmented observer rudder dynamic estimation precision is low, can not meet the need of fault detection and isolation problems.

\section{Problem Description}

The aircraft equation as follows:

$$
\left\{\begin{array}{l}
\dot{\boldsymbol{x}}(t)=\boldsymbol{A x}(t)+\boldsymbol{B} \boldsymbol{\delta}(t) \\
\boldsymbol{y}(t)=\boldsymbol{c x}(t)
\end{array}\right.
$$


Where $\boldsymbol{A} \in \boldsymbol{R}^{n \times n}, \boldsymbol{B} \in \boldsymbol{R}^{n \times m}, \boldsymbol{c} \in \boldsymbol{R}^{n \times n}, \boldsymbol{x}(t)$ is observable part of the system state, $\boldsymbol{\delta}(t)$ is the actual rudder deflection angle.

Actuator is first-order kinetic model inertia. when no fault control relationship between actual instruction and the rudder deflection angle is as follows:

$$
\left\{\begin{array}{l}
\boldsymbol{\delta}(t)=f(\boldsymbol{\delta}) \boldsymbol{u}(t) \\
\dot{\boldsymbol{\delta}}(t)=\boldsymbol{B}_{\omega}(\boldsymbol{u}(t)-\boldsymbol{\delta}(t))
\end{array}\right.
$$

Where $f(\boldsymbol{\delta})$ is the transfer function of actuator, $\boldsymbol{B}_{\omega} \in \boldsymbol{R}^{m \times m}$ is transfer gain.

For rapid fault diagnosis system (1), a simple method is to mature rudder augmented to the state, the use of augmented observer were estimated rudder deflection. However, augmented observation itself there are some key issues unresolved, that dynamic changes augmented state vector estimate poor performance, not even track this dynamic change. Using observer for fault diagnosis, an important and obvious fact is that rapid fault isolation on the premise that the observer status include fast dynamic response to changes in estimation and tracking with high accuracy. Therefore, how to design a new augmented so that it is not only stable observer error is small, and can quickly and accurately estimate the state of the dynamic response to meet the requirements for rapid fault diagnosis is the most important issue to be addressed in this article.

For the above problems, this paper consider the speed and accuracy requirements. A method for high-precision adaptive compensation observer is proposed to estimated dynamic rudder deflection. And a new adaptive threshold is designed for rapid fault detection. The limited memory least squares method used reset the initial value, which to achieve a rapid identification of mutation parameters.

\section{The Adaptive Compensation Observer}

\section{Augmented Observer}

In the case of actuator failure, the control inputs $\boldsymbol{\delta}(t)$ can't be acquired, the input observer ideas are present that the control input is augmented into the state, if the estimated outputs $\hat{y}(t)$ track the outputs, using the mature method of state observer, the estimated states $y(t)$ and the actual inputs $\hat{\boldsymbol{\delta}}(t)$ can be estimated at the same time.

The augmented state is given as follows:

$$
X=\left[\begin{array}{ll}
x & \delta
\end{array}\right]^{T}
$$

Then the system equation (1) are described by the following state space equations:

$$
\begin{aligned}
\dot{\boldsymbol{X}} & =\overline{\boldsymbol{A}} \boldsymbol{X}(t)+\overline{\boldsymbol{B}} \dot{\boldsymbol{\delta}}(t) \\
\boldsymbol{y} & =\overline{\boldsymbol{C}} \boldsymbol{X}(t)
\end{aligned}
$$

where $\overline{\boldsymbol{A}}=\left[\begin{array}{cc}\boldsymbol{A} & \boldsymbol{B} \\ \boldsymbol{0}_{m \times n} & \boldsymbol{0}_{m \times m}\end{array}\right], \overline{\boldsymbol{B}}=\left[\begin{array}{c}\boldsymbol{0}_{n \times m} \\ \boldsymbol{I}_{m \times m}\end{array}\right], \overline{\boldsymbol{C}}=\left[\begin{array}{c}\boldsymbol{C} \\ \boldsymbol{0}_{n \times m}\end{array}\right]^{T}$, and the $\dot{\boldsymbol{\delta}}(t)$ is the first-order derivative of the $\boldsymbol{\delta}(t)$.

The augmented state of observer can be defined as:

$$
\hat{\boldsymbol{X}}=\left[\begin{array}{ll}
\hat{\boldsymbol{x}} & \hat{\boldsymbol{\delta}}
\end{array}\right]^{T}
$$

Where the estimates quantities are represents by the $(\wedge)$. So the system equations of the observer are obtained as:

$$
\begin{aligned}
\dot{\hat{X}} & =\bar{A} \hat{X}+\boldsymbol{L} \bar{C}(X-\hat{X}) \\
\hat{y} & =\bar{C} \hat{X}
\end{aligned}
$$

Where the $L \in \boldsymbol{R}^{(n+m) \times r}$ is the gain of the observer, of which the errors is 


$$
\boldsymbol{e}=\boldsymbol{X}-\hat{\boldsymbol{X}}
$$

Referring to the equation (4), (5) and (6), the dynamic value of observation error is given as

$$
\dot{\boldsymbol{e}}=(\overline{\boldsymbol{A}}-\boldsymbol{L} \overline{\boldsymbol{C}}) \boldsymbol{e}+\overline{\boldsymbol{B}} \dot{\boldsymbol{\delta}}
$$

Suppose that the $(\bar{A}, \bar{C})$ is observable, using the bilinear quadratic adjustor method, the gain $L$ can be obtained as follows:

$$
L=P_{0} \bar{C}^{T} R_{0}^{-1}
$$

Where the $\boldsymbol{P}_{\boldsymbol{0}}$ is the solution of the Riccati equation.

$$
\boldsymbol{O}=\overline{\boldsymbol{A}} \boldsymbol{P}_{0}+\boldsymbol{P}_{0} \overline{\boldsymbol{A}}^{T}+\boldsymbol{Q}_{0}-\boldsymbol{P}_{0} \overline{\boldsymbol{C}}^{T} \boldsymbol{R}_{0}^{-1} \overline{\boldsymbol{C}} \boldsymbol{P}_{0}
$$

Where the $\boldsymbol{Q}_{0}$ is the positive semi-definite weighting matrix, $\boldsymbol{R}_{0}$ is the positive definite weighting matrix. When $\dot{\delta}$ tends to 0 , the estimate error also is approaching 0 .

\section{Adaptive Dynamic Compensation}

Although the system inputs can be estimated by the augmented observer, for the rapid response systems of control surfaces, the augmented observer still have some questions as follows:

1)The state error of the system is essentially regarded as the foundation for the augmented observer, which often has high precision for state estimation, but the estimation accuracy of the control input is low.

2) The augmented observer cannot track the rapid changes of the control surfaces' dynamic response.

The formula (8) shows that the dynamic error $\dot{\boldsymbol{e}}$ is determined by $(\overline{\boldsymbol{A}}-\boldsymbol{L} \overline{\boldsymbol{C}})$ and $\dot{\boldsymbol{\delta}}$. When the actuator moves and the $\dot{\delta}$ is unequal to zero, there will be a large estimation error.

That problems will seriously affect the accuracy of the fault checking and the fault isolation precision. Whether the input observer can be applied depends on how to improve the input estimation accuracy. An adaptive dynamic compensation method is used to solve these problems, given by equation (11):

$$
\dot{\hat{\boldsymbol{x}}}(t)=\boldsymbol{A} \hat{\boldsymbol{x}}(t)+\boldsymbol{B} \hat{\boldsymbol{\delta}}(t)
$$

According to the first-order kinetic model of the actuator, using the estimated values of the control surfaces to replace the true values, the estimated virtual residual can be represented by $\tilde{\boldsymbol{\delta}}(\tilde{\boldsymbol{\delta}}=\boldsymbol{u}-\hat{\boldsymbol{\delta}})$, then the formula (12) can be given by:

$$
\dot{\boldsymbol{\delta}}=\boldsymbol{B}_{\omega} \tilde{\boldsymbol{\delta}}
$$

Let $\tilde{\boldsymbol{x}}=\boldsymbol{x}-\hat{\boldsymbol{x}}$, so

$$
\boldsymbol{B}(\boldsymbol{\delta}-\hat{\boldsymbol{\delta}})=\dot{\tilde{\boldsymbol{x}}}-\boldsymbol{A} \tilde{\boldsymbol{x}}
$$

When the dynamic variety is uniquely considered, then $\boldsymbol{u}-\hat{\boldsymbol{\delta}} \approx \boldsymbol{\delta}-\hat{\boldsymbol{\delta}}$, then

$$
\dot{\boldsymbol{\delta}}=\boldsymbol{B}_{\omega} \tilde{\boldsymbol{\delta}} \approx \boldsymbol{B}_{\omega} \boldsymbol{B}^{\dagger}(\dot{\tilde{\boldsymbol{x}}}-\boldsymbol{A} \tilde{\boldsymbol{x}})
$$

Where $\boldsymbol{B}^{\dagger}=\boldsymbol{B}^{T}\left(\boldsymbol{B B}^{T}\right)^{-1}$ is the pseudo inverse of the matrix $\boldsymbol{B}$, supposed that in the moment $0 \rightarrow t_{0}$, the control surfaces changed, so the dynamic values can be described :

$$
\begin{aligned}
\Delta \boldsymbol{\delta} & =\int_{0}^{t_{0}} \dot{\boldsymbol{\delta}} d \tau \approx \int_{0}^{t_{0}} \boldsymbol{B}_{\omega} \boldsymbol{B}^{\dagger}(\dot{\tilde{\boldsymbol{x}}}-\boldsymbol{A} \tilde{\boldsymbol{x}}) d \tau \\
& =\boldsymbol{B}_{\omega} \boldsymbol{B}^{\dagger} \tilde{\boldsymbol{x}}-\int_{0}^{t_{0}} \boldsymbol{B}_{\omega} \boldsymbol{B}^{\dagger} \boldsymbol{A} \tilde{\boldsymbol{x}} d \tau
\end{aligned}
$$


Ignoring the slow variation item $\int_{0}^{t_{0}} \boldsymbol{B}_{\omega} \boldsymbol{B}^{\dagger} \boldsymbol{A} \tilde{\boldsymbol{x}} d \tau$, the adaptive dynamic compensation equation can be designed to compensate the control surfaces' fast dynamic variety, described as:

$$
\Delta \boldsymbol{\delta}=\boldsymbol{K} \boldsymbol{B}^{\dagger} \tilde{\boldsymbol{x}}
$$

Where $\boldsymbol{K}=\operatorname{diag}\left\{k_{1} \quad k_{2} \quad \cdots \quad k_{m}\right\},\left(k_{i}>0\right)$ is compensation gain, which is confirmed by the estimated residual error and the augmented observer's pseudo error, given as: $\tilde{\boldsymbol{x}}=\boldsymbol{x}-\hat{\boldsymbol{x}}$. The adaptive dynamic compensation idea is that the error of control input estimation is essentially caused by the inaccurate of state estimation for the augmented observer, so it is considered that translate the observable state estimation error into the input estimation error, and then compensate the error into the input estimation. In virtue of fast of state estimation, the compensated input estimation also can quickly track control surfaces response.

The observation equation can be described as:

$$
\begin{aligned}
\dot{\hat{\boldsymbol{X}}} & =\overline{\boldsymbol{A}} \hat{\boldsymbol{X}}+\boldsymbol{L} \overline{\boldsymbol{C}}(\boldsymbol{X}-\hat{\boldsymbol{X}})+\overline{\boldsymbol{B}} \Delta \dot{\boldsymbol{\delta}}(t) \\
\hat{\boldsymbol{y}} & =\overline{\boldsymbol{C}} \hat{\boldsymbol{X}}
\end{aligned}
$$

Then the dynamic observation error value is

$$
\dot{\overline{\boldsymbol{e}}}=(\overline{\boldsymbol{A}}-\boldsymbol{L} \overline{\boldsymbol{C}}) \overline{\boldsymbol{e}}+\overline{\boldsymbol{B}}(\dot{\boldsymbol{\delta}}-\Delta \dot{\boldsymbol{\delta}})
$$

Where the $\overline{\boldsymbol{e}}$ is the new observation error, though equation(18) improved cannot avoid the estimated error when the actuator makes a movement, it can achieve the fast dynamic track, which can improve the accuracy of the estimated input. when $\dot{\boldsymbol{\delta}} \rightarrow \boldsymbol{0}, \tilde{\boldsymbol{x}} \rightarrow \boldsymbol{0}, \Delta \boldsymbol{\delta} \rightarrow \boldsymbol{0}, \overline{\boldsymbol{e}} \rightarrow \boldsymbol{0}$.

The control surfaces estimation $\hat{\boldsymbol{\delta}}$ can be achieved from the augmented state estimation $\hat{\boldsymbol{X}}$, so the virtual residual error is

$$
\boldsymbol{r}(t)=\boldsymbol{u}(t)-\hat{\boldsymbol{\delta}}(t)
$$

Where the actual control surface deflection error is unknown, so $r(t)$ is virtual residual error.

\section{Observer Numerical Simulation}

Certain control surfaces of aircraft is used. Selected 4 groups of rudder control $\boldsymbol{u}=\left[u_{c}, u_{r e}, u_{l e}, u_{r}\right]^{T}$, which represent the canards, right elevator aileron, left elevator ailerons, rudder angle. $\boldsymbol{x}=[\alpha, \beta, p, q, r]^{T}$ is state variables, to represent the angle of corner speed, yaw, roll, pitch and yaw rates. $r(t)=[\alpha, \beta, p]$ is reference instruction. Altitude is $H=3000 m$ and Mach is $M a=0.22$. Linear model of plane as follows:

$$
\begin{gathered}
\boldsymbol{A}=\left[\begin{array}{ccccc}
-0.6896 & 0 & 0 & 0.9777 & 0 \\
0 & -0.1585 & 0.1376 & 0 & -0.9812 \\
0 & -14.1883 & -1.2681 & 0 & 0.5192 \\
3.5436 & 0 & 0 & -0.6681 & 0 \\
0 & 0.7722 & -0.0868 & 0 & -0.2821
\end{array}\right] \quad \boldsymbol{B}=\left[\begin{array}{cccc}
-0.0292 & -0.1250 & -0.1250 & 0 \\
0 & 0.01451 & -0.0145 & 0.0373 \\
0 & -8.2155 & 8.2155 & 2.5171 \\
2.9175 & -2.6711 & -2.6711 & 0 \\
0 & -0.6254 & 0.6254 & -1.5633
\end{array}\right] \\
f\left(\delta_{i}\right)=20 /(s+20) \\
\boldsymbol{\delta}_{p \max }=\left[\begin{array}{llll}
25 & 30 & 30 & 30
\end{array}\right]^{\mathrm{T}}, \\
\boldsymbol{\delta}_{p \min }=-\left[\begin{array}{llll}
55 & 30 & 30 & 30
\end{array}\right]^{\mathrm{T}}, \\
\boldsymbol{\delta}_{R \max }=-\boldsymbol{\delta}_{R \min }=\left[\begin{array}{llll}
50 & 50 & 50 & 50
\end{array}\right]^{\mathrm{T}} .
\end{gathered}
$$


Assuming the $6^{\circ}$ angle of attack is applied to the instruction of the aircraft in 3 to 30 seconds. the $100 \%$ roll angle rate of application instructions in 10 to 40 seconds. The side slip angle reference instruction is always $0^{\circ}$. Canard $40 \%$ damage when a fault occurs 15 seconds. Left elevons $40 \%$ damage in 25 seconds. Fault simulation step size is 0.01 seconds, the actuator white noise variance is $0.025\left(^{\circ}\right)^{2}$. Closed-loop system state response process shown in Figure 1:
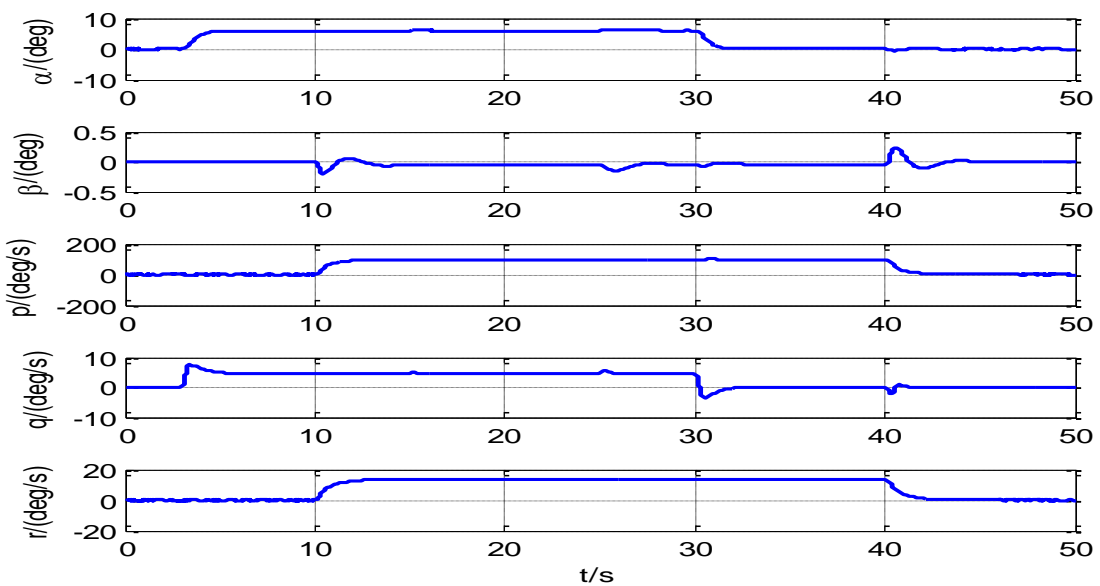

Figure 1. Flight states response of closed-roll system

As Figure 1 shows that, due to the presence of a fault-tolerant control law, so that the command signal on flight status tracking, to ensure system stability, control surface control instructions and observer estimates shown in Figure 2:
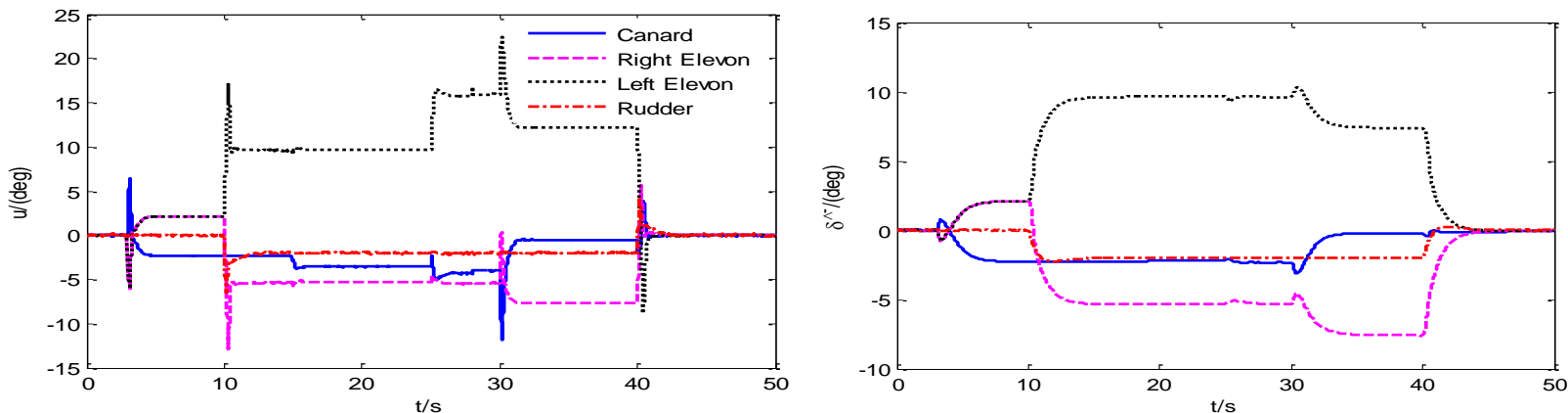

(a) The control command

(b) The estimated value by Augmented Observer
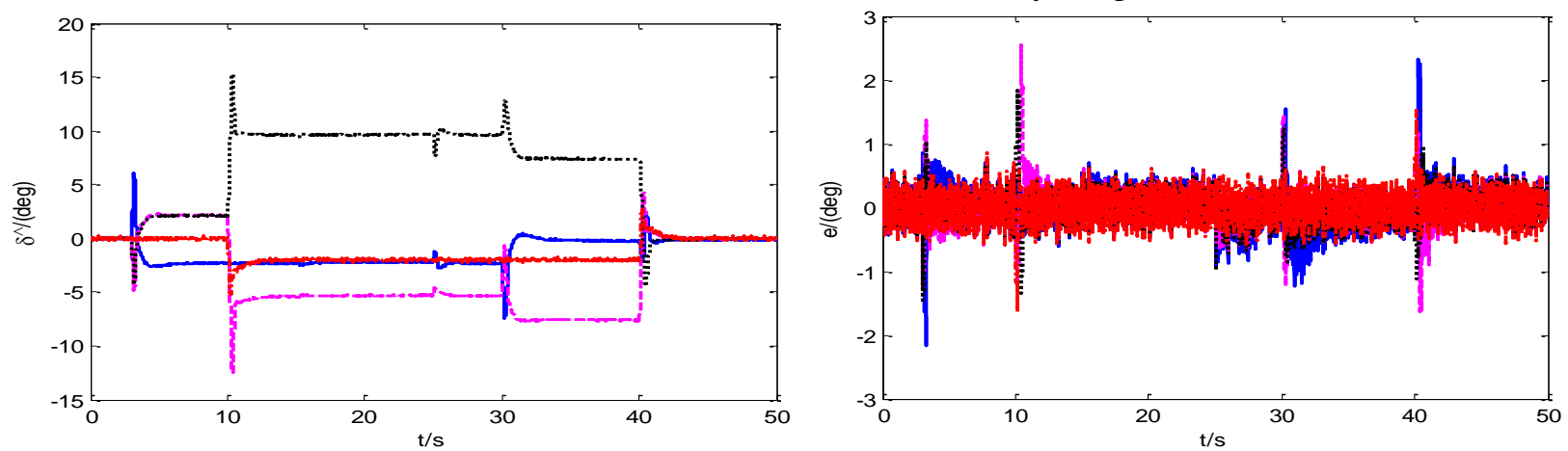

(c) The estimated value by Adaptive Observer

(d) The error between (a) and (c)

Figure 2. Control surface command and observer estimates

By Figure 2 (a) and (b) known, the general observer augmented estimation accuracy is better at the rudder steady. But when the rudder rapid changes, tracking slow, error is large. Figure 2 (b) and (c) comparison shows that adaptive dynamic compensation after the observer, which can fast track rudder changes, while maintaining the steady precision. Deflection from Figure 2 (d) adaptive 
compensation observer error and the actual rudder shows adaptive compensation observer observing the steady state error is less than 1 degree, the dynamic error of less than 3 degrees higher accuracy.

\section{Conclusions}

This paper presents a fast fault detection and isolation method for a multi-aircraft rudder control surface damage, the following conclusions:

1) adaptive compensation method can be directly estimated observer aircraft rudder deflection, adaptive compensation method greatly improves the observer on the rudder fast dynamic tracking capabilities, to achieve a precise estimate of the rudder deflection.

2) adaptive threshold designed to reduce leakage alarm rate and false alarm rate, failure to achieve a rapid alert.

\section{References}

1. Chen FuYang, Jiang Bing. Direct Sslt-repairing Control for Aircraft[M]. Beijing: National Defense Industry Press, 2014: 1-5.

2. Zhou DongHua, Ding X. Theory and Applications of Fault Tolerant Control[J]. Acta Automatica Sinica, 2000, 26(6): 787-797.

3. Chen ZongJi, Zhang RuLin, Zhang Ping et al. Flight Control: Challenges and Opportunities[J]. Acta Automatica Sinica, 2013, 39(6):703-710

4. Girish Chowdhary, Wesley M. DeBusk and Eric N. Johnson. Real-Time System Identification of a Small Multi-Engine Aircraft with Structural Damage[C]. AIAA Infotech @ Aerospace 2010, AIAA 2010-3472.

5. Eugene A. Morelli. Real-Time Parameter Estimation in the Frequency Domain[J]. Journal of Guidance, Control and Dynamics, 2000, 23(5):812-818.

6. Tang We, Qiao Qian, Shi ZhongKe. On the Choice of Parameter Constraint for Frequency domain Least Squares Identification[J], Acta Aeronautica et Astronautica Sinica. 2012, 33(12):2253-2260.

7. Huang Chengtao,Wang LiXI. On-line Fault Diagnosis for Control Surfaces of Multi-control Effector Flying Wings[J]. Acta Aeronautica et Astronautica Sinica. 2011,32(1):58-66.

8. Su Haoqin, Song Shujie, Deng Jianhua. A Better On-Line Identification Algorithm with Impairment of Aircraft Control Surfaces Considered[J]. Journal of Northwestern Polytechnical University, 2005, 23(3):316-320

9. Matthew C. Ruschmann and N. Eva Wu. Actuator Fault Diagnosis Using Two-Stage Extended Kalman Filters[C]. AIAA Guidance, Navigation, and Control Conference, AIAA 2010-7703.

10. Y.Han, S. Oh and B.Choi et al. Fault Detection and Identification of Aircraft Control Surface Using Adaptive Observer and Input Bias Estimator[J]. IET Control Theory and Applications, 2012, 6(10):1367-1387

11. Guillaume J.J. Ducard. Fault-tolerant Flight Control and Guidance Systems[M]. Chen ZiLi, Xie ZhiGang, translated. Beijing: National Defense Industry Press, 2012: 43-84.

12. Hu Shousong, Guo Wei, Zhang Defa. Methods of Structure Fualt Detection and Reconfiguration of Self-repairing Control Law for a Fighter[J]. Acta Aeronautica et Astronautica Sinica. 1998,19(6):674-677 
13. Zhang Ping, Chen ZongJi. Fault Detection Filter for the Control Surface Failures of Aircraft[J]. Acta Aeronautica et Astronautica Sinica. 1999,20(4):371-373

14. Li Qing, Shen Chunlin, Guo Suofeng. Detection and Isolation of Control Surface Effectiveness Faitures[J]. Acta Aeronautica et Astronautica Sinica. 1997, 18(6):693-697 